\title{
Inhibition of Wound Epidermis Formation via Full Skin Flap Surgery During Axolotl Limb Regeneration
}

\author{
Stephanie Tsai $^{1,2}$ \\ ${ }^{1}$ Department of Stem Cell and Regenerative Biology, Harvard University ${ }^{2}$ Department of Molecular and Cellular Biology, Harvard University
}

\section{Corresponding Author}

Stephanie Tsai

stsai@mgh.harvard.edu

\section{Citation}

Tsai, S. Inhibition of Wound Epidermis Formation via Full Skin Flap Surgery During Axolotl Limb Regeneration. J. Vis. Exp. (160), e61522, doi:10.3791/61522 (2020).

\section{Date Published}

June 24,2020

\section{DOI}

$10.3791 / 61522$

URL

jove.com/video/61522

\section{Abstract}

Classical experiments in salamander regenerative biology over the last century have long established that the wound epidermis is a crucial signaling structure that forms rapidly post-amputation and is required for limb regeneration. However, methods to study its precise function at the molecular level over the last decades have been limited due to a paucity of precise functional techniques and genomic information available in salamander model systems. Excitingly, the recent plethora of sequencing technologies coupled with the release of various salamander genomes and the advent of functional genetic testing methods, including CRISPR, makes it possible to revisit these foundational experiments at unprecedented molecular resolution. Here, I describe how to perform the classically developed full skin flap (FSF) surgery in adult axolotls in order to inhibit wound epidermis formation immediately following amputation. The wound epidermis normally forms via distal migration of epithelial cells in the skin proximal to the amputation plane to seal off the wound from the outside environment. The surgery entails immediately suturing full thickness skin (which includes both epidermal and dermal layers) over the amputation plane to hinder epithelial cell migration and contact with the underlying damaged mesenchymal tissues. Successful surgeries result in the inhibition of blastema formation and limb regeneration. By combining this surgery method with contemporary downstream molecular and functional analyses, researchers can begin to uncover the molecular underpinnings of wound epidermis function and biology during limb regeneration.

\section{Introduction}

Since Lazzaro Spallanzani reported it in $1768^{1}$, salamander limb regeneration has been one of the most well-studied natural regenerative phenomenon that has enamored biologists for centuries. Successful limb regeneration hinges on the formation, outgrowth, and subsequent patterning of an undifferentiated cellular structure known as the blastema. Researchers have made significant strides in understanding the cellular composition of the blastema as well as which 
supportive tissues and cell types are necessary for its

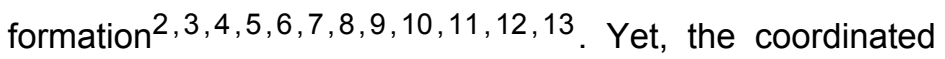
signaling mechanisms between different tissues and cell types that lead to the initiation of blastema formation remain poorly understood.

A key requirement for successful blastema formation and regeneration is the wound epidermis, a transient and specialized epithelium that covers the amputation plane within 12 hours post-amputation ${ }^{10}$. Following amputation, epithelial cells from the intact skin proximal to the injury rapidly migrate over the amputation plane to form a thin wound epithelium ${ }^{14}$. As the blastema forms in the following weeks, the early wound epidermis develops into a thicker epithelial signaling structure called the apical epithelial cap $(A E C)^{15}$. While normal full thickness skin contains both an epithelial and dermal layer separated by a basal lamina, the wound epidermis/AEC consists only of an epithelial layer and lacks a basal lamina ${ }^{16,17}$. The absence of the basal lamina and dermis allows for direct contact between the wound epithelial cells and the underlying tissues, which facilitates bidirectional signaling between the two compartments that is critical for both blastema formation and maintenance 17,18 .

Classical experimental studies devised various innovative surgical methods to probe wound epidermis/AEC function and necessity via inhibiting its formation. These methods included suturing ${ }^{19}$ or grafting full thickness skin 20,21 over the amputation plane, immediately suturing the amputated limb into the body cavity ${ }^{22}$, and continuous daily removal or irradiation of the early wound epidermis and $A E C^{23,24}$. Altogether, these experiments not only established the importance of the wound epidermis/AEC, but also further determined its roles in early tissue histolysis as well as maintaining progenitor cell proliferation and blastemal outgrowth $^{13}$ throughout regeneration.

However, these previous studies were largely limited to histological staining as well as tritiated thymidine pulses to track cell proliferation. In fact, revisiting these classical experiments with modern sequencing technologies and functional techniques in salamanders has only recently been done and has led to the discovery of additional roles for the wound epidermis in modulating inflammation and ECM degradation/deposition during early stages of regeneration ${ }^{25}$. With the release of various salamander genome and transcriptome sequences $26,27,28,29,30,31,32,33,34$, as well as the burgeoning number of functional methods available in salamander species ${ }^{11,35,36,37,38}$, researchers are now well-positioned to begin to unravel the molecular mechanisms driving wound epidermis formation, function, and $A E C$ development.

Unfortunately, several of these classical methods used to inhibit wound epidermis formation are technically challenging, presenting difficulties for reproducibility between biological replicates in the same experiment. For instance, maintaining skin grafts can be challenging as grafts may eventually fall off the host limb and removal of the wound epidermis/AEC daily is difficult without damaging the underlying tissues. Furthermore, suturing the amputated limb into the body cavity is challenging and also requires additional injury at the site of insertion. On the other hand, suturing full thickness skin immediately over the amputation plane is relatively simple, technically reproducible, and introduces minimal tissue damage. This full skin flap (FSF) surgical method was previously developed by Anthony Mescher in 1976 in adult newts (Notophthalmus viridiscens). He demonstrated that the FSF surgery inhibited wound epidermis formation and 
function by prohibiting both epithelial cell migration over the amputation plane and direct contact between epithelial cells and the underlying tissues.

Here, this surgical procedure is shown step-by-step using the axolotl limb. Coupled with modern day molecular and sequencing technologies, this technique may prove to be very helpful for researchers to deepen our understanding of wound epidermis/AEC formation and function during limb regeneration.

\section{Protocol}

All animal experiments were performed in accordance with IACUC (Protocol \#: 11-32) and AAALAC guidelines at Harvard University.

\section{Preparing solutions and setup for anesthesia and recovery}

1. Prepare fresh $0.1 \%$ tricaine solution for anesthesia and $0.5 \%$ sulfamerazine sodium salt solution for recovery. Make the solutions using water suitable for axolotl husbandry ${ }^{37}$ according to approved IACUC protocols at the relevant research institution (modified Holtfreter's solution, for instance). Make sure that the solutions are well-mixed and that enough volume is prepared in order to submerge the entire axolotl.

1. To prepare $0.1 \%$ tricaine solution, mix $1 \mathrm{~g}$ of tricaine and $1 \mathrm{~g}$ of sodium bicarbonate with $1 \mathrm{~L}$ of water. The solution can be scaled up according to this recipe.

2. To prepare $0.5 \%$ sulfamerazine sodium salt solution, mix $5 \mathrm{~g}$ of sulfamerazine sodium salt with $1 \mathrm{~L}$ of water. The solution can be scaled up according to this recipe. Sulfamerazine solution is an anti-biotic that will prevent bacterial infection during surgical recovery.

2. Sanitize the surgical area by spraying it down with Clidox-S or $70 \%$ ethanol. Sterilize surgical tools (forceps, dissecting scissors, spring scissors) by autoclaving. If performing multiple surgeries, make sure to sterilize the surgical tools with a hot bead sterilizer between animals.

3. To set up the recovery area, place a $15 \mathrm{~cm}$ Petri dish or any container that will fit the axolotl on top of a bucket filled with wet ice. Fill the Petri dish with a low level of $0.5 \%$ sulfamerazine sodium salt solution, enough such that the axolotl would not be fully submerged. The recovery on ice post-surgery will slow the animal's movement while it awakens from anesthesia, allowing the sutured area to heal relatively undisturbed.

NOTE: This setup can be customized by researchers depending on what materials they have available.

\section{Performing the full skin flap surgical procedure}

1. Anesthetize the axolotl by submersing it in a container of $0.1 \%$ tricaine solution. This will take approximately 15-20 minutes. Ensure that the axolotl is indeed fully anesthetized by performing a tail pinch. If there is no response from the axolotl, proceed with the surgery.

NOTE: Use older, larger axolotls for this surgery (at least $15 \mathrm{~cm}$ in size). Make sure that the axolotl remains well hydrated throughout the surgery by wetting the skin periodically with axolotl system water using a plastic pipette. Make sure to aseptically prepare the surgical site by irrigating the area with sterile PBS prior to surgery. The animal should also be placed on a sterile surgical drape for the procedure. 
2. Perform a limb amputation at the distal end of the zeugopodial skeletal elements using the dissecting scissors (Figure 1.1).

3. Using the spring scissors, make a small incision (approximately $2 \mathrm{~mm}$ ) on the ventral portion of the skin (Figure 1.2).

4. Using the forceps, carefully peel back the skin to approximately the midline of the zeugopodial skeletal elements, exposing the underlying limb tissues (muscle, bone, etc.) (Figure 1.3). Make sure not to damage the skin. See note after step 2.8.

5. Amputate the exposed underlying limb tissues at the midline of the zeugopod using surgical scissors (Figure 1.4).

6. Push back the muscle tissue with the surgical scissors and trim the exposed bone.

NOTE: This is necessary to ensure improved healing and also to increase the success of the surgery as protruding bone can be jagged against the sutured flap and disrupt the integrity of an intact skin flap later on.

7. Using the forceps, carefully pull the extra full thickness skin over the amputation plane to cover the exposed underlying tissues and suture in place by connecting with the ventral full thickness skin (Figure 1.5).

8. Suture the remaining right and left sides of the skin flap into the underlying ventral portions of intact skin. This can be done by either suturing the sides of the flap in a "criss-cross" manner (recommended) (Figure 1.6-1.9), or simply suturing straight into the ventral skin. Use the forceps and curved spring scissors for suturing. Make sure no exposed underlying tissues can be seen and that sutures are tied tight (knotted at least three times).
NOTE: It is critical that the intact skin is not damaged in steps 2.4, 2.7-2.8. We have found that damage to the full thickness skin has been correlated with unsuccessful surgeries, as the areas of damage may still form a small wound epidermis. If possible, try to use a duller pair of forceps when handing the full thickness skin flap.

9. Perform an amputation on the contralateral limb (optional internal animal control) by amputating it at mid-zeugopod level with surgical scissors. Push back the muscle tissue with surgical scissors and trim the exposed bone.

NOTE: An internal contralateral limb control can be done to better assess the success of the surgery during step 4 in the same animal. However, amputation of the same limb in a separate animal can also be used to serve as a control.

\section{Post-operative recovery and care}

1. Once the surgery is complete, place a Kimwipe or sterile paper towel at the bottom of the container or Petri dish to wet it. Place the animal into the container on wet ice and gently wrap the exposed ends of the Kimwipe or paper towel around the top of the animal to keep it wellhydrated with sulfamerazine solution. Leave on wet ice for 30 minutes to 1 hour to ensure minimal movement during recovery from anesthesia.

2. Place the animal into a static housing container with $0.5 \%$ sulfamerazine solution. Axolotls must remain in this solution for the first 24 hours in order to prevent infection.

3. Place the axolotl into normal system water and monitor health daily. Ensure that no sutures fall out each day as this can result in a small wound epidermis forming which will confound results.

NOTE: Make sure that the housing container has ample room for the axolotl to move around and minimize the 
chances that the sutured limb on the axolotl can come into contact with the sides of the container. This will help to ensure that the sutures stay in place, especially during the first week post-surgery.

\section{Assessing success of the surgery under a stereo microscope}

NOTE: We recommend checking animals under a stereo microscope at least once a week to assess the integrity of the full skin flap and success of the surgery.

1. Anesthetize the axolotl in $0.1 \%$ tricaine as in Step 2.1 . Make sure there is ample room in the container for the axolotl to move around.

2. If inspecting during the first two weeks post-surgery, inspect the sutured limb using a stereomicroscope to make sure no sutures have popped out and that a clear thin wound epidermis is not visible anywhere. If inspecting on the third week post-surgery or later, make sure a blastema has not formed and compare with how the normal control amputated limb (either from the same animal or a different animal) has progressed during regeneration (i.e., whether a blastema has formed).

3. When done, return the axolotl to normal system water and husbandry conditions.

\section{Representative Results}

This surgical protocol will allow for the complete inhibition of wound epidermis formation (Figure 1) and ultimately, limb regeneration. A successful surgery results in no blastema formation in approximately 2-3 weeks depending on the size of the animal, while control regenerating limbs should form a blastema normally.

Researchers should inspect the sutured limb by naked eye every 2-3 days to make sure that the sutures have not popped out and that a blastema is not forming. If one or more of the sutures pop out, a wound epidermis can still form resulting in either a small or large blastema and an unsuccessful surgery (Figure 2). Additionally, researchers should inspect the sutured limb at least once every week under a stereomicroscope to make sure that a thin wound epidermis is not evident anywhere on the amputation surface. For comparison, researchers should also examine the control regenerating limb which should have a wound epidermis over the amputation plane and form a blastema over 2-3 weeks. The wound epidermis will appear thin and clear, while the normal skin will appear more opaque and pale pink (almost white), light yellow, or dark green in leucistic, albino, or wildtype axolotls, respectively.

If researchers wish to collect tissue prior to the blastema formation stages at 2-3 weeks, they should inspect the sutured limbs prior to sample collection to make sure the sutures remained in place and that a small wound epidermis did not form. Additionally, sectioning sagitally through the sutured limb tissue and performing histological analyses at any time point can also verify the presence of the dermis from the full skin flap encircling the entire amputation plane and the absence of a wound epidermis (Figure 3). 


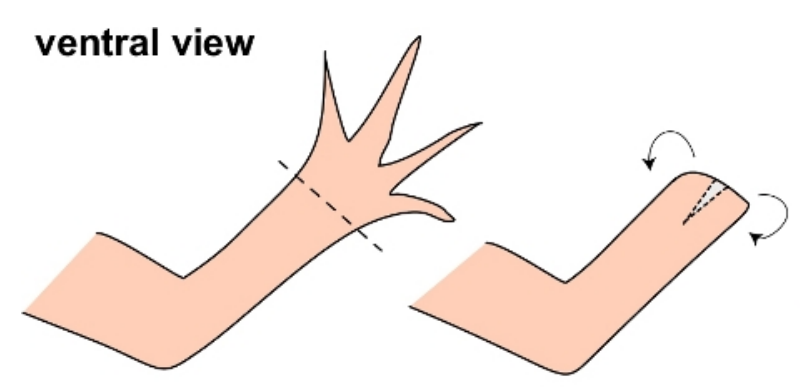

1

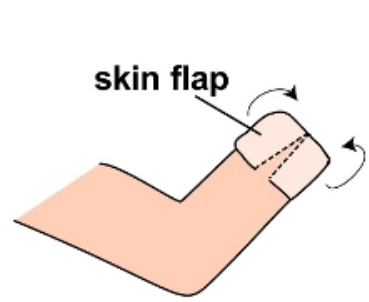

4

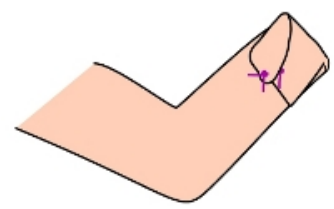

7
2

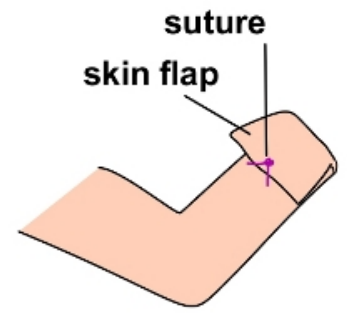

5

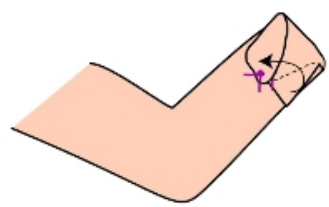

8

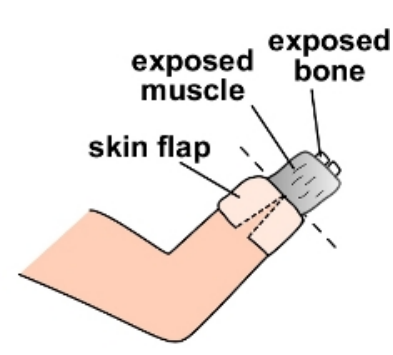

3

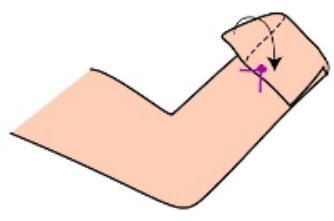

6

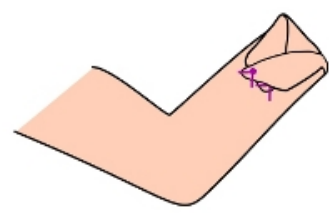

9

Figure 1: Schematic of the steps of the full skin flap surgery.

The steps of the protocol are numbered and diagrammed here. The dotted lines denote the planes of amputation at steps 1 and 3 of the protocol. Please click here to view a larger version of this figure. 

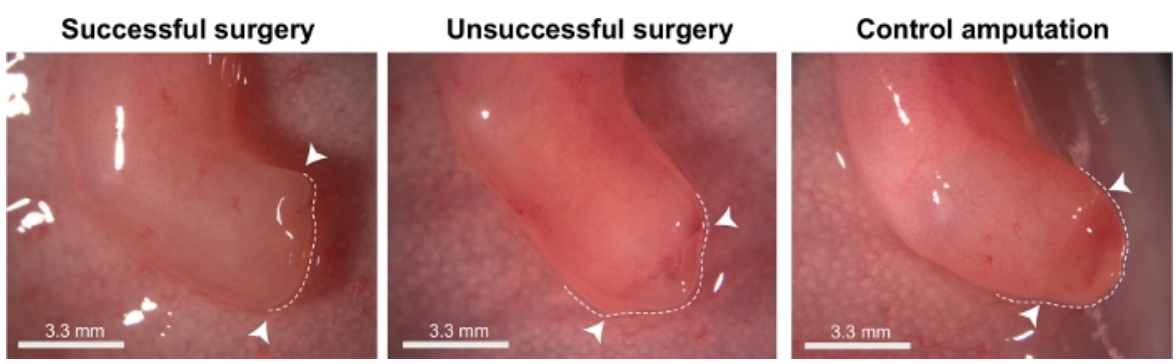

Figure 2: Examples of successful and unsuccessful full skin flap surgeries.

Representative brightfield image of a limb that has undergone a successful surgery (left), an unsuccessful surgery (right), and a control regenerating limb (no surgery) at 25 days post-amputation (dpa). The successful surgery has a flat amputation plane where the full skin flap was sutured over, whereas the unsuccessful surgery has a small blastema developing.

Arrowheads denote the amputation plane and white dotted lines are there to aid visualization of the absence of a blastema in the successful surgery and presence of blastemas in the unsuccessful surgery and control regenerating limbs. Please click here to view a larger version of this figure. 

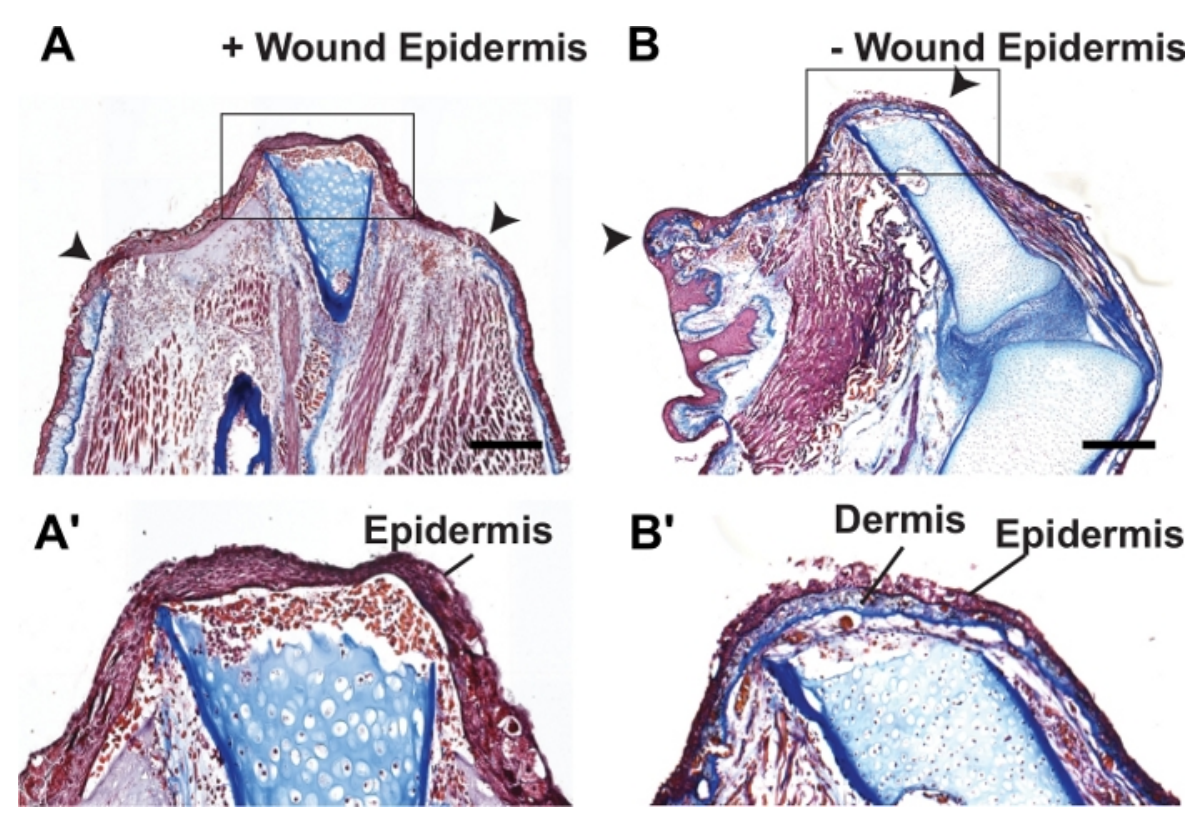

Figure 3: Histological staining of normal regenerating and FSF sutured limbs.

(A-B') Representative brightfield images of picro-mallory stained sections from regenerating (A-A') and sutured axolotl limbs (B-B') at 7 dpa. Insets in A and B are shown in A' and B', respectively. The collagen-heavy dermal layer lines and covers the entire amputation plane in sutured limbs. Amputation plane is denoted by arrowheads in A-B. Scale bars represent 500 $\mu \mathrm{m}$. This figure was adapted from Tsai et al. ${ }^{25}$. Please click here to view a larger version of this figure.

\section{Discussion}

This article describes a protocol for performing full skin flap surgeries in axolotl limbs to inhibit wound epidermis formation. While this surgery is relatively simple and technically reproducible compared to other methods of inhibiting wound epidermis formation, there are several critical steps that can impact the success of the surgery. First, when pulling the intact full skin flap over the exposed underlying tissues, it is paramount that the full thickness skin not be damaged in any way. Damage to the skin flap can still lead to the formation of a small wound epidermis, which can result in a small blastema-like outgrowth. Second, ensure sutures do not fall out during post-operative care as this can also lead to the formation of a small wound epidermis.
To this point, minimizing the potential contact between the sutured limb and any surfaces is important, especially during the first week post-surgery. Several ways to prevent this entail housing and anesthetizing the axolotl in a large enough container such that the axolotl has plenty of room to move around post-surgery.

This surgery also has several limitations. Perhaps the most notable is that the success of surgeries can only be assessed in two ways: using the dissecting scope during the first two weeks of surgery to search for an absence of a wound epidermis and/or checking whether a blastema forms within 3 weeks. While these methods are effective, they are relatively low throughput. The development of future transgenic reporter axolotls for wound epidermis-specific 
markers may aid in quicker screening for successful versus unsuccessful surgeries. Furthermore, this surgery is more difficult to perform on younger animals as the intact skin is more fragile. Using sub-adult or adult axolotls is thus recommended.

While this surgery was originally developed in $N$. viridiscens $^{19}$, it has been easily adapted for axolotls 25,39 and can likely be applied to other salamander species as well. In sum, applying this technique to future limb regenerative studies will empower researchers to both develop more tools to address wound epidermis biology and identify the underlying mechanisms driving its function in initiating blastema formation.

\section{Disclosures}

The authors have nothing to disclose.

\section{Acknowledgments}

The author thanks Doug for his constant encouragement and unwavering support, as well as the members of the Melton lab for their helpful feedback and comments on the manuscript. The author would also like to thank the Harvard Office of Animal Resources (OAR) for their dedicated animal care.

\section{References}

1. Spallanzani, L. Prodromo Di Un'opera Da Imprimersi Sopra Le Riproduzioni Animali. Nella Stamperia di Giovanni Montanari, 1768, (1768).

2. Gerber, T. et al. Single-cell analysis uncovers convergence of cell identities during axolotl limb regeneration. Science. (2018).

3. Leigh, N. D. et al. Transcriptomic landscape of the blastema niche in regenerating adult axolotl limbs at single-cell resolution. Nature Communications. 9 (1), 5153 (2018).

4. Kragl, M. et al. Cells keep a memory of their tissue origin during axolotl limb regeneration. Nature. 460 (7251), 60-65 (2009).

5. McCusker, C., Bryant, S. V., Gardiner, D. M. The axolotl limb blastema: cellular and molecular mechanisms driving blastema formation and limb regeneration in tetrapods. Regeneration (Oxford). 2 (2), 54-71 (2015).

6. Endo, T., Bryant, S. V., Gardiner, D. M. A stepwise model system for limb regeneration. Developmental Biology. 270 (1), 135-145 (2004).

7. Tsai, S. L. The molecular interplay between progenitors and immune cells in tissue regeneration and homeostasis. Journal of Immunology and Regenerative Medicine. 7100024 (2020).

8. Godwin, J. W., Pinto, A. R., Rosenthal, N. A. Macrophages are required for adult salamander limb regeneration. Proceedings of the National Academy of Sciences of the United States of America. 110 (23), 9415-9420 (2013).

9. Tanaka, E. M. The molecular and cellular choreography of appendage regeneration. Cell. 165 (7), 1598-1608 (2016).

10. Campbell, L. J., Crews, C. M. Wound epidermis formation and function in urodele amphibian limb regeneration. Cellular and Molecular Life Sciences. 65 (1), 73-79 (2008).

11. Fei, J. F. et al. Efficient gene knockin in axolotl and its use to test the role of satellite cells in limb regeneration. Proceedings of the National Academy of Sciences of the United States of America. 114 (47), 12501-12506 (2017). 
12. Sandoval-Guzman, T. et al. Fundamental differences in dedifferentiation and stem cell recruitment during skeletal muscle regeneration in two salamander species. Cell Stem Cell. 14 (2), 174-187 (2014).

13. Tassava, R. A., Mescher, A. L. The roles of injury, nerves, and the wound epidermis during the initiation of amphibian limb regeneration. Differentiation. 4 (1), 23-24 (1975).

14. Hay, E. D., Fischman, D. A. Origin of the blastema in regenerating limbs of the newt Triturus viridescens. An autoradiographic study using tritiated thymidine to follow cell proliferation and migration. Developmental Biology. 3 26-59 (1961).

15. Christensen, R. N., Tassava, R. A. Apical epithelial cap morphology and fibronectin gene expression in regenerating axolotl limbs. Developmental Dynamics. 217 (2), 216-224 (2000).

16. Repesh, L. A., Oberpriller, J. C. Scanning electron microscopy of epidermal cell migration in wound healing during limb regeneration in the adult newt, Notophthalmus viridescens. American Journal of Anatomy. 151 (4), 539-555 (1978).

17. Neufeld, D. A., Day, F. A., Settles, H. E. Stabilizing role of the basement membrane and dermal fibers during newt limb regeneration. Anatomical Record. 245 (1), 122-127 (1996).

18. Singer, M., Saltpeter, M. M. in Growth in Living Systems (ed M.X. Zarrow) Basic Books, New York. (1961).

19. Mescher, A. L. Effects on adult newt limb regeneration of partial and complete skin flaps over the amputation surface. Journal of Experimental Zoology. 195 (1), 117-128 (1976).
20. Tassava, R. A., Garling, D. J. Regenerative responses in larval axolotl limbs with skin grafts over the amputation surface. Journal of Experimental Zoology. 208 (1), 97-110 (1979).

21. Tornier, G. Der Kampf der Gewebe im Regeneratbei Begunsiigung der Hautregeneralion. Arch. Entwmech. 22 348-352 (1906).

22. Goss, R. J. Regenerative inhibition following limb amputation and immediate insertion into the body cavity. Anatomical Record. 126 (1), 15-27 (1956).

23. Thornton, C. S. The effect of apical cap removal on limb regeneration in Amblystoma larvae. Journal of Experimental Zoology. 134 (2), 357-381 (1957).

24. Thornton, C. S. The inhibition of limb regeneration in urodele larvae by localized irradiation with ultraviolet light. Journal of Experimental Zoology. 137 (1), 153-179 (1958).

25. Tsai, S. L., Baselga-Garriga, C., Melton, D. A. Midkine is a dual regulator of wound epidermis development and inflammation during the initiation of limb regeneration. Elife. 9 (2020).

26. Nowoshilow, S. et al. The axolotl genome and the evolution of key tissue formation regulators. Nature. 554 (7690), 50-55 (2018).

27. Elewa, A. et al. Reading and editing the Pleurodeles waltl genome reveals novel features of tetrapod regeneration. Nature Communications. 8 (1), 2286 (2017).

28. Bryant, D. M. et al. A Tissue-Mapped Axolotl De Novo Transcriptome Enables Identification of Limb Regeneration Factors. Cell Reports. 18 (3), 762-776 (2017). 
29. Looso, M. et al. A de novo assembly of the newt transcriptome combined with proteomic validation identifies new protein families expressed during tissue regeneration. Genome Biology. 14 (2), R16 (2013).

30. Abdullayev, I., Kirkham, M., Bjorklund, A. K., Simon, A., Sandberg, R. A reference transcriptome and inferred proteome for the salamander Notophthalmus viridescens. Experimental Cell Research. 319 (8), 1187-1197 (2013).

31. Burns, J. A., Zhang, H., Hill, E., Kim, E., Kerney, R. Transcriptome analysis illuminates the nature of the intracellular interaction in a vertebrate-algal symbiosis. Elife. 6 (2017).

32. Nakamura, K. et al. A transcriptome for the study of early processes of retinal regeneration in the adult newt, Cynops pyrrhogaster. PLoS One. 9 (10), e109831 (2014).

33. Smith, J. J. et al. A chromosome-scale assembly of the axolotl genome. Genome Research. 29 (2), 317-324 (2019).

34. Arenas Gomez, C. M., Woodcock, R. M., Smith, J. J., Voss, S. R., Delgado, J. P. Using transcriptomics to enable a plethodontid salamander (Bolitoglossa ramosi) for limb regeneration research. BMC Genomics. 19 (704) (2018).

35. Fei, J. F. et al. Application and optimization of CRISPR-Cas9-mediated genome engineering in axolotl (Ambystoma mexicanum). Nature Protocols. 13 (12), 2908-2943 (2018).

36. Flowers, G. P., Timberlake, A. T., McLean, K. C., Monaghan, J. R., Crews, C. M. Highly efficient targeted mutagenesis in axolotl using Cas9 RNA-guided nuclease. Development. 141 (10), 2165-2171 (2014).

37. Khattak, S. et al. Optimized axolotl (Ambystoma mexicanum) husbandry, breeding, metamorphosis, transgenesis and tamoxifen-mediated recombination. Nature Protocols. 9 (3), 529-540 (2014).

38. Joven, A., Elewa, A., Simon, A. Model systems for regeneration: salamanders. Development. 146 (14) (2019).

39. Johnson, K., Bateman, J., DiTommaso, T., Wong, A. Y., Whited, J. L. Systemic cell cycle activation is induced following complex tissue injury in axolotl. Developmental Biology. 433 (2), 461-472 (2018). 